\title{
Histoire de village, parcours de musicien
}

\section{Bernard Lortat-Jacob}

\section{(2) OpenEdition \\ Journals}

Édition électronique

URL : http://journals.openedition.org/ethnomusicologie/601

ISSN : 2235-7688

Éditeur

ADEM - Ateliers d'ethnomusicologie

Édition imprimée

Date de publication : 1 novembre 2002

Pagination : 17-31

ISSN : 1662-372X

Référence électronique

Bernard Lortat-Jacob, « Histoire de village, parcours de musicien », Cahiers d'ethnomusicologie [En ligne], 15 | 2002, mis en ligne le 11 janvier 2012, consulté le 03 mai 2019. URL : http:// journals.openedition.org/ethnomusicologie/601

Ce document a été généré automatiquement le 3 mai 2019.

Tous droits réservés 


\title{
Histoire de village, parcours de musicien
}

\author{
Bernard Lortat-Jacob
}

\section{Tore et Totore}

1 Les villages du centre de la Sardaigne ont une caractéristique : leurs habitants s'appellent souvent Salvatore - en abrégé Totore. Ceux du nord sont à cet égard différents; les Salvatore y sont aussi nombreux, mais leur prénom ne s'abrège jamais en « Totore », mais seulement en « Tore».

2 La Sardaigne vit au rythme de ces nuances. Elles sont essentielles puisque, conduit comme je le suis à couvrir à la fois le nord et le centre de l'île, il m'arrive de me tromper de façon scandaleuse dans ce système d'abréviations et de dire "Tore» pour "Totore», ou l'inverse. «Il a vraiment l'esprit ailleurs aujourd'hui », dit-on de moi. Mais cet « ailleurs » n'est pas n'importe où : il est chez le voisin, tout simplement. A noter que le voisin c'est l'étranger. Le seul véritable étranger, dont on se démarque toujours, pour rappeler qu'on est différent de lui et pour être sans doute davantage soi-même.

Précisons qu'au village, ce grand nombre de Totore ou de Tore ne gêne pas vraiment la communication. Il n'est jamais nécessaire de désigner celui de qui l'on parle autrement que par son prénom abrégé. Le sujet de la conversation se charge du reste : histoires de brebis égarées ou volées? c'est de Totore Lai qu'il s'agit [il est «vigile communal » et toujours très informé de tout ce qui peut arriver aux douze mille brebis du village ${ }^{1}$; d'accordéon diatonique? C'est naturellement de Totore Chessa qu'il est question. Et c'est justement de lui que je vais parler².

4 Soyons précis : il y a vingt ans - lorsque nous nous sommes connus - ce dernier ne s'appelait pas Totore, mais Totoreddu. Je pense - sans pouvoir l'affirmer complètement - que ce diminutif, il le tenait de sa mère. Une mère sarde d'excellence. Et, pour elle, ce petit suffixe « $e d d u$ » final ne voulait pas dire : «Voyez comme il est petit !», mais plutôt «Regardez comme il est grand pour son âge !» Cette grandeur se voyait déjà à son autorité de jeune homme et de musicien. Et la mère a abandonné ce diminutif il y a 
une quinzaine d'années, lorsqu'il n'avait plus lieu d'être. En effet, à 25 ans, Totore était en passe de devenir le $\mathrm{n}^{\circ} 1$ des accordéonistes de Sardaigne. Tout le monde pressentait cette gloire... mais sa mère dut l'anticiper de quelques années: elle lui donna son prénom actuel pour couper le cordon ombilical - ce qui intervient toujours fort tard en Sardaigne - mais surtout pour faire de son fils un "Totore " à part entière. De sorte qu'aujourd'hui, lorsque dans les trois cent cinquante villages de Sardaigne, on entend le nom de Totore à propos d'accordéon et de danse, on sait de quel Totore il est question. C'est de Salvatore Chessa, d'Irgoli [Baronia, Province de Nuoro]. Les autres - les très jeunes ou les obscurs - ont encore besoin de leur nom de famille pour exister ${ }^{3}$.

Revenons à cette autorité musicale : elle est, chez Totore, d'abord et surtout rythmique. Il joue d'un diatonique à huit basses. Le Paolo Soprani de ses débuts était particulièrement lourd. Avec une machine à soufflet de ce poids, il fallait une grande force pour imprimer à la danse son indispensable légèreté. L'accordéon sarde n'a véritablement rien à voir avec celui d'un André Verchuren. Pour en jouer, il faut des bras d'athlète. Ces bras, Totore les acquit en pratiquant son premier métier - maçon - tandis que ses doigts durent souffrir des mauvais traitements du marteau, de la rugosité du ciment et des «blocchetti». Mais personne n'eut jamais à se plaindre des dégâts que durent occasionner ces travaux de maçonnerie sur les doigts de Totore ; les autres, pas plus que lui-même.

Fig. 1 : Totore jouant d'un accordéon chromatique de sa collection.

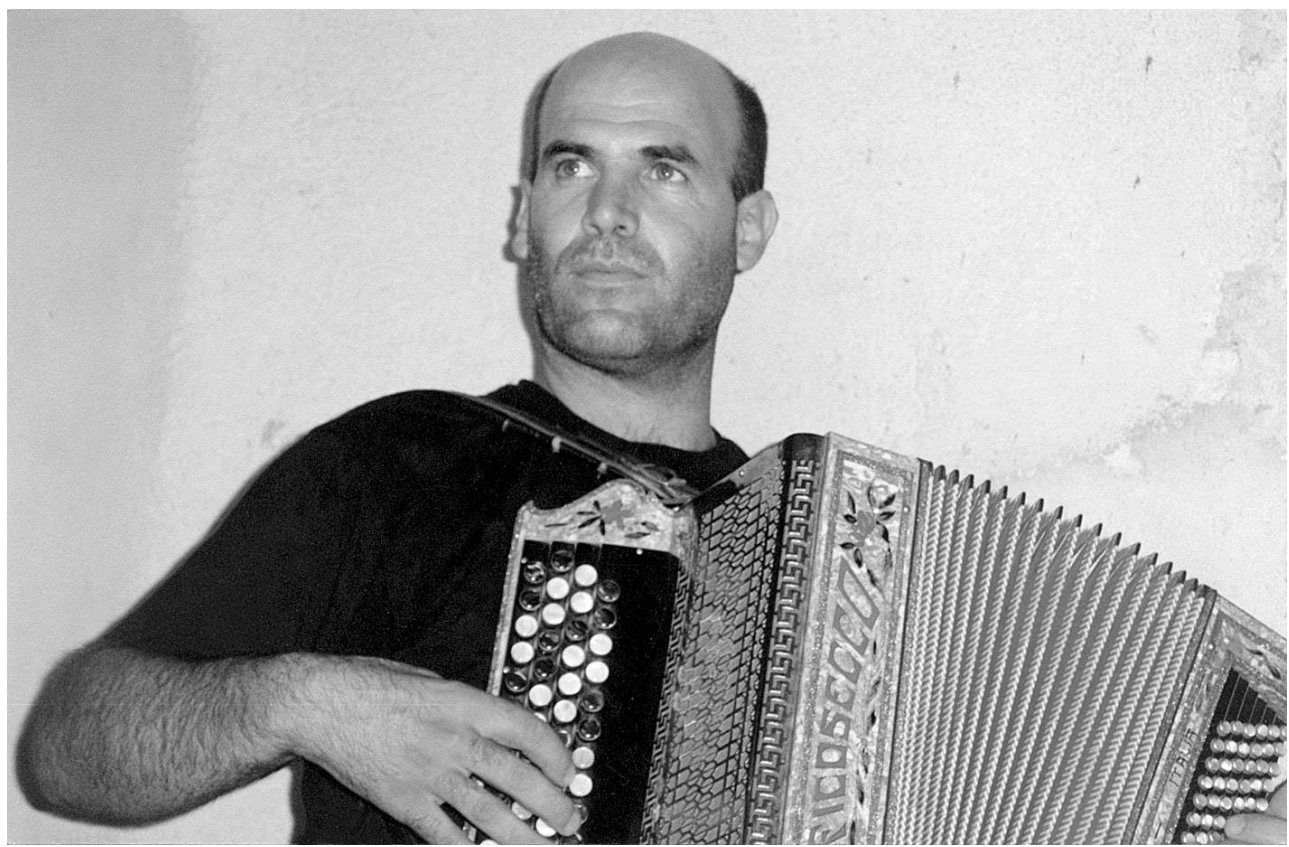

Photo : Bernard Lortat-Jacob, 1995

Précisons que, pour un musicien - et un musicien de danse en particulier - le rythme se confond avec l'autorité. Il lui en faut beaucoup pour assujettir totalement les danseurs à sa propre conception de la danse et pour que ces derniers, in fine,lui confient le mouvement de leurs corps. Cette maîtrise réclame un sens aigu de l'anticipation : un bon suonatore doit savoir lire les attentes de ceux qu'il dirige du haut de son petit podium et les interpréter, notamment pour faire alterner comme il le faut les pas glissés ou sautés ( passu bassu ou altu). Combiner l'art et l'acrobatie en somme. Ça, c'est pour le ballu in piazza , « la danse en place » [c'est-à-dire sur la place], où s'exécute le ballu tundu (danse en 
rond), le ballu sarde par excellence que, depuis plus de vingt ans maintenant, Totore anime durant les fêtes en été et qui prolongent, la nuit, la célébration rituelle d'un Saint Patron local. De nos jours, cette danse communautaire, impliquant la participation de tous, est certes encore pratiquée durant les fêtes, mais elle est de plus en plus rare. Or, tous les musicien le savent et le disent - du moins lorsqu'ils connaissent leur Sardaigne : « un accordéoniste et une place, cela suffit pour faire la fête !»

7 Si j'aime tout particulièrement le ballu in piazza, c'est pour des raisons strictement professionnelles et ethnomusicales. Raisons que Totore conteste par principe - « Toi, tu es un homme de musée, tu n'y comprends rien! » me reproche-t-il (entre autre). Cela ne m'empêche pas de penser que rien n'est plus beau qu'un village s'offrant lui-même en représentation. Tandis qu'il donne une image de sa propre présence, chacun voit l'autre, l'observe, le rencontre, le perd de vue momentanément, le retrouve, etc. Quant à notre ami musicien, s'il aime cette danse, c'est aussi pour lui-même, lorsqu'il devient, le temps de quelques heures (ou parfois de quelques minutes), le roi de la place et le véritable prince du village. Pas question, dit-il, que chacun danse chacun de son côté [per conto suo] ou de façon désordonnée " comme les brebis dans la campagne ». La place n'est pas une discothèque : la compostezza - la «bonne tenue ", qui est aussi une façon de tenir son rang avec dignité - est de rigueur.

Bien danser est la moindre des choses. En fait, à Irgoli, tout le monde sait le faire et le village est connu dans toute la Sardaigne pour la qualité de sa danse. Mais la marque d'un grand danseur tient dans ce qu'il sait exprimer par sa façon de danser : il est tantôt léger, tantôt grave, sévère ou rigolard, courtois ou grossier, distant ou attentif. Attentif aux autres, bien sûr. Et dans un jeu d'incessantes interactions, l'ensemble doit faire cercle, quitte à ce que, par moment, le cercle se brise pour laisser s'échapper quelques couples en direction de son centre. Instants spectaculaires toujours très contrôlés.

En somme, pour des raisons symétriques et opposées à celles de Totore, je suis d'accord avec lui. Sauf sur un point, car j'ai tendance à penser que les brebis sardes ont toujours une façon très harmonieuse de s'assembler, comme si le troupeau avait une conscience de lui-même.

De nos jours cependant, la Sardaigne paesana [paysanne et villageoise] vit de moins en moins au rythme de ce ballu in piazza. Elle a désormais ses gruppi folk, groupes folkloriques, qui, certes, n'ont rien d'aussi abominables que la plupart des nôtres, mais qui sont désormais omniprésents. Portée par eux sur un petit podium dominant la place, la danse devient alors une représentation. Sous une forte amplification, et destinée à un public désormais immobile, elle s'exprime à travers des interprétations musicales et chorégraphiques standardisées (tout gruppo folk a son maître de danse - en principe, un bon danseur). C'est le règne également du costume traditionnel qui n'est plus guère porté qu'à cette occasion et dans ce contexte ${ }^{4}$. En une vingtaine d'années, la musique sarde, à Irgoli comme ailleurs, est progressivement sortie de son rang de musique communautaire pour entrer dans le monde du spectacle.

Retraçons les étapes de cette évolution.

\section{Années soixante-dix}

Totore n'a pas été seulement spectateur d'un changement qui concerne son village et toute la Sardaigne ; il en a été aussi l'un des principaux acteurs. 

surnommé «ziu Tonareddu »- le « petit homme de Tonara », un village de Barbagia dont sa famille était probablement originaire - qui mourut d'ailleurs récemment. Il avait deux danses à son répertoire: celle d'Irgoli - le ballu "brincu», une danse «sautée » très simple, mais de belle facture, composée essentiellement de deux formules musicales alternantes - une pour le pas normal [passu bassu], une pour le pas «sauté » [altu]. Ces deux formules suffisaient pour faire danser tout le pays durant des soirées entières. Le deuxième ballu était une danse standard - celle «des autres » en quelque sorte, qu'il jouait de temps en temps «cosi, per cambiare» ("pour changer un peu»). Ailleurs en Sardaigne, c'était la même situation. Un grand suonatore comme Mondo Vercellino ne jouait pas autre chose que les danses de son village de Bono, situé dans le Goceano; et maintenant qu'il est un peu âgé, il n'a guère pris le temps, ni sans doute éprouvé le besoin, d'élargir beaucoup son répertoire.

Totore n'avait guère plus de seize ans lorsque dans son village d'Irgoli, bien connu par la qualité de sa danse et le caractère allegro de ses habitants, s'est créé un gruppo folk. C'est l'époque où, comme il le dit, les gruppi folk poussaient sur l'île "comme des champignons $»^{6}$. Au départ, celui d'Irgoli n'était pas fondamentalement centré sur la production de spectacles. Il était formé d'un groupe relativement informel de jeunes gens qui se rassemblaient plusieurs fois par semaine pour pratiquer la musique et danser.

Fig. 2 : Ballu in piazza à Irgoli.

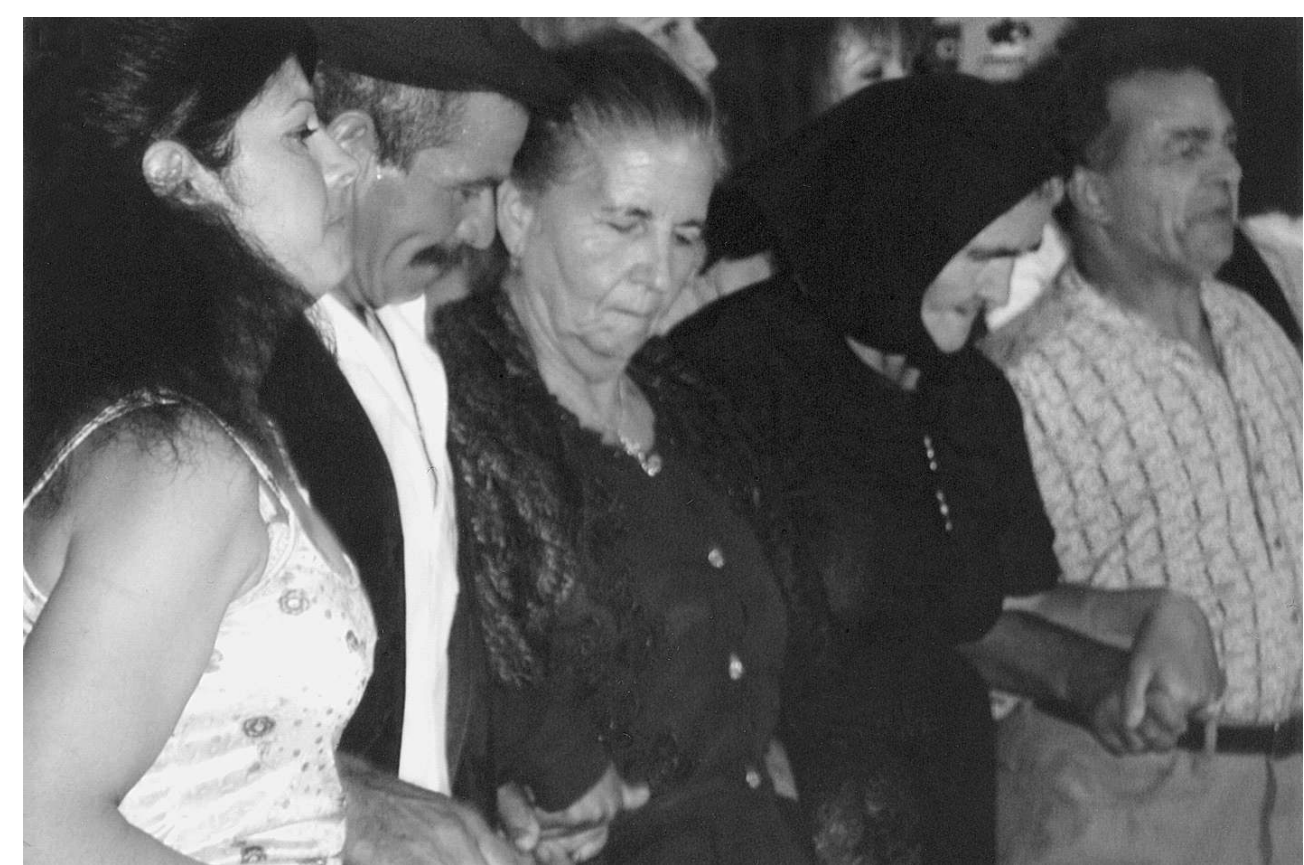

Photo : Bernard Lortat-Jacob

Dans les années soixante-dix, Totore était déjà le pilier de ce petit conservatoire de musique populaire, qui avait aussi pour fonction d'être une sorte de « maison des jeunes » et se tenait dans un bar périphérique - le "bar Vacca", peu visité en dehors de ces soirées - un bar d'assez larges dimensions et dont le propriétaire était lui-même chanteur. Tout le monde ou presque s'y rendait, non seulement pour danser au son de l'accordéon de Totore, mais aussi pour chanter en chœur ou avec l'accompagnement de la guitare et pour boire un verre, manger saucisse et fromage, blaguer et parler. En 
Sardaigne, la musique est impratiquable en dehors de ce contexte. La première ambition du groupe d'alors était... d'être groupe, et de vivre comme tel. Et si, aujourd'hui, les hommes et femmes de 50 ans montrent encore à Irgoli autant de compétences dans l'art du chant et de la danse, c'est à la fréquentation du « bar Vacca » qu'ils la doivent.

Par l'éclatante joie qui le caractérisait autant que par le talent de ceux qui l'animent, ce gruppo eut vite fait d'occuper le devant de la scène, en Sardaigne, et occasionnellement à l'étranger. Il remporta le «premier prix de Folklore " à Goritza en 1979. J'étais à Irgoli lorsqu'un membre du gruppo en fit l'annonce téléphonique en appelant le bar central du village. Ce fut immédiatement un concert de klaxons de toutes les Vespas, car à Irgoli, il n'y avait à cette époque plus guère de chevaux et pas encore beaucoup de voitures, seulement des Vespas.

\section{Années quatre-vingts}

17 Au milieu des années quatre-vingts, le gruppo connut de violentes dissensions. De ce genre de dissensions très fréquentes en Sardaigne qui procèdent d'un mécanisme bien réglé et qui intéressent directement l'anthropologie ${ }^{7}$. Totore s'est disputé avec un autre Totore, vigile communal, lui aussi très dynamique, mais qui ne connaissait rien à l'accordéon. Il était bon danseur et jouait de la guimbarde. Cette dispute aboutit à un clivage, puis à un éclatement prévisible. Alors notre Totore accordéonniste s'allia à un autre Totore, chanteur-guitariste et frère du propriétaire du "bar Vacca», en laissant le Totore danseur créer son propre gruppo. Bref, on passa d'un gruppo à deux gruppi.

Cette scission eut un effet très négatif sur l'ambiance du village. Les deux gruppi pensaient de plus en plus à assurer leur carrière. Les sorties se multiplièrent avec des tournées à l'étranger, qui servaient surtout à asseoir le prestige d'un groupe au détriment de l'autre. La danse fit les frais de cette situation nouvelle car le spectacle, un peu forcé, en donne désormais une expression figée. Son tempo augmente année après année pour servir une virtuosité qui n'est pas toujours du meilleur goût et qui a pour effet de retirer au ballu une partie de sa grâce. La discorde prend corps et, progressivement, les groupes des deux Totore créent deux traditions distinctes, qui se différencient par leur conception de la danse. Certes, les pas qu'ils exécutent sont les mêmes, mais Totore-l'accordéoniste préfère éviter les chorégraphies empruntées (dans tous les sens du terme); il fait danser «ses » danseurs sous une forme simple, voisine de celle pratiquée dans le ballu in piazza, tandis que l'autre Totore marque une nette préférence pour l'exhibition: danses en cercle, en chaîne, en cercle de nouveau, en étoile, etc. Celui qu'on appelle désormais non plus le gruppo d'Irgoli, mais le gruppo de Totore - ex-Totoreddu - ne manque pas de rafler de nombreux prix. Dans la maison maternelle, la salle de réception, que personne ne fréquente en fait, puisque, comme partout en Sardaigne, c'est toujours dans la cuisine que l'on vit, est couverte de trophées. 
Fig. 3 : Au bar des pastori, Irgoli



Photo : Bernard Lortat-Jacob, 1997

\section{Années quatre-vingt dix}

l'accordéon diatonique - qui le mobilise, lui et son gruppo, chaque année en août, et où il invite tous les accordéonistes de Sardaigne, ainsi que d'autres en provenance de quelques obscures provinces d'Europe. L'opération est culturelle et politique : à peine créé, le festival devient "fête du pays » et Irgoli le centre de la danse et de l'accordéon en Sardaigne. Quant à Totore, il est désormais le grand fédérateur de musiciens locaux qui entretiennent à l'ordinaire des rapports de jalousie, ou qui souvent se connaissent mal, du fait du morcellement culturel de l'île. Les Irgolais ont vite fait de s'enorgueillir d'une telle 
initiative : ils deviennent juges, notamment de la suprématie de leur ballu et de leur suonatore. Au fond, ils n'en demandaient pas plus.

4.Enfin, pour l'éditeur continental Robi Droli, il réalise en 1996 un disque dont il décide lui-même de la composition et du maquettage. Pour quelques morceaux, il se fait accompagner par les musiciens et chanteurs insulaires les plus connus et les plus représentatifs. La publication bénéficie de la caution de mon ami et collègue Francesco Giannattasio, qui signe le texte de présentation du disque. La notice est bilingue : pour la première fois, l'anglais apporte un appui promotionnel à une production strictement locale. La pochette représente l'accordéon déplié comme une fleur et tenu en l'air. Totore lui-même ne figure qu'au verso de cette métaphore aérienne, souriant comme il se doit.

Fig. 4 : La culture des pastori : danse sur scène à Irgoli.



Photo : Bernard Lortat-Jacob, 2000

Aucune référence explicite à la Sardaigne dans ce disque - ni fêtes, ni danseurs, ni même paysages - hormis l'attitude particulièrement figée de Totore qui n'est pas sans rappeler celle des portraits photographiques d'autrefois, montés dans de vieux cadres et que l'on trouve encore dans toutes les maisons. La pochette du disque est composée autour du nom de Totore Chessa, lequel n'apparait pas moins de trois fois. Le titre de l'album est écrit en très petit: organittos [néologisme sarde crée à partir du mot italien organetto désignant l'accordéon diatonique]. Quant au son du disque, il n'est pas mauvais seulement un peu trop tiède, adouci et sensiblement réverbéré.

Avant que le disque ne sorte, Francesco et moi discutons longuement avec Totore de son intention de signer toutes les musiques présentes dans le disque alors que celui-ci est exclusivement composé de danses traditionnelles villageoises et sardes, bien entendu très bien exécutées. Initiative un peu scandaleuse, selon nous et dont Luigi Lai - un grand joueur de launeddas - a été l'initiateur pour ses disques à lui. Face à notre réprobation 
étonnée, Totore semble un peu reculer. L'édition finale aboutit à un compromis assez confus où il apparaît que chaque pièce est identifiée : a) par un nom qui rappelle de façon plus ou moins claire l'origine de la musique (Baronia, Costera, Supramontes [sic]); b) par un deuxième nom renvoyant au type de danse (passu torrau, dillu, etc.; enfin c) toutes portent le nom de Totore Chessa, ainsi que celui de l'éditeur. Une chatte n'y retrouverait pas ses petits. Je ne sais pas si la S.I.A.E. - la société italienne de droits d'auteur - y retrouve les siens 8 .

Ce disque connaît un certain succès commercial et marque une date dans la carrière de Totore : alors que, durant les années précédentes, il s'était partiellement affranchi des liens particulièrement forts qui le rattachaient à son village d'origine, le voici qui s'affranchit désormais de la Sardaigne tout entière. Elle n'est pas citée. Il y a là, à la fois une forme d'usurpation - les musiciens qui jouaient ces danses avant Totore ne sont pas cités - et un transfert de propriété. Les danses ne sont plus celles de Sardaigne, mais les siennes.

L'ambiguïté demeure toutefois car, désormais, si Totore est invité partout, c'est pour sa "Sardité ». S'il joue aussi bien - il le sait pertinemment - c'est parce qu'il n'a jamais cessé de conduire le ballu in piazza. C'est ainsi qu'on ne verra jamais Totore faire une faute de rythme ou se tromper dans la dynamique d'un accent. Seul l'art de la danse et celui de faire danser lui apportent ces qualités musicales.

Et pendant ce temps-là, les choses s'aggravent sérieusement à Irgoli. Pour des raisons obscures, et qui doivent le rester, le village entre dans un cycle de violence dont la scission du gruppo folk n'était qu'une illustration métaphorique. Une vendetta aux origines troubles se réveille. Meurtres en série, (une dizaine de morts en cinq ans, ce qui est considérable pour un village de quelque 2000 habitants), mais aussi bétail volé, chevaux assassinés, voitures brûlées. Surtout celles des employés communaux - et donc du Totore-vigile. Et les tracteurs aussi. Et les portes et fenêtres de la poste et de la mairie régulièrement criblées de balles. Intimidations de toute nature. A leurs jeunes enfants, qui savent encore à peine marcher, les mères recommandent: "Surtout, si tu vois quelque chose, tu ne dis rien!» L'omertà s'apprend tôt. Cette omertà arrange tout le monde, dans le fond: même les carabinieri venus du continent, qui tournent sans cesse dans le village en faisant semblant de conduire une enquête, mais sans prendre le risque de beaucoup sortir de leurs $4 \times 4$. D'ailleurs, à cinq heures du soir, le portail de la gendarmerie est fermé - horaires de fonctionnaires oblige, alors que, bien entendu, c'est après cinq heures du soir que les choses se passent - les "Cinco de la tarde ", écrivait Lorca...

La danse, à nouveau, fait les frais de ce climat, cette fois-ci franchement délétère. On danse avec de moins en moins de cœur et d'adresse. Et paradoxalement, pas plus tard que l'an dernièr, et par un acte volontariste qui peut d'ailleurs s'expliquer, le village voit naître un troisième gruppo, qui prétend insuffler un nouveau dynamisme, mais occasionne surtout un nouveau clivage, bien peu nécessaire celui-ci. Et si les trois gruppi dansent tous les mêmes danses - celles du pays - ils ne le font jamais ensemble. Par les temps qui courent, le ballu in piazza, lorsqu'il se pratique encore, notamment lors de la fête de San Michele ou de San Nicola, ne ressemble plus à grand chose. 


\section{Trois Totore en un...}

vanter son village pour sa musique tout autant que pour la qualité de ses habitants, la
production de ses saucisses et la beauté de ses melons [trois spécialités du pays, en effet]. Il est alors exclusivement musicien de (son) village. A ses tout débuts, la danse du pays reposait encore sur les frêles épaules de «ziu Tonareddu ». Totore n'a pas même la trace enregistrée du ballu de son prédécesseur qu'il a entendu durant toute son enfance. Il n'a jamais été son élève. D'ailleurs « ziu Tonareddu » n'aurait jamais eu l'idée d'enseigner à quiconque cette danse qu'il se contentait de jouer, à la façon d'un artisan consciencieux et infatigable. Mais c'est bien cette danse que Totore, dès le départ, joue à la perfection et sans doute mieux que « ziu Tonareddu » lui-même. Cette première étape de sa carrière, il la franchit en démontrant qu'il n'est pas un émule discret et de seconde classe. Il faut reconnaître qu'elle n'était pas très difficile à mémoriser [ce qui ne veut pas dire pour autant facile à jouer]. Très vite, il se l'approprie, modifiant sensiblement la forme sans altérer le fond; il lui ajoute quelques formules brillantes et personnelles dont la perfection formelle et la puissance dynamique plurent immédiatement aux danseurs. En Sardaigne, il faut d'abord être reconnu par son village pour espérer exister en dehors de lui. Car c'est lui qui vous donne vos lettres de noblesse ainsi qu'une authentique légitimité. Et si tous les villageois sont attentifs à la réputation de leur paese, c'est parce qu'elle a des incidences immédiates sur la leur. Totore le sait bien. Aujourd'hui comme par le passé, il reste toujours fier de ses origines « baroniese » [de Baronia].

Durant la deuxième période, Totore embrasse plus largement le territoire insulaire. En moins d'une dizaine d'années, il devient musicien régional. La fréquentation des différentes places où il est invité pour animer le ballu ou accompagner des gruppi est à la base de sa deuxième formation. "C'est mon Université à moi, dit-il... C'est là que j'apprends les mille et une façons qu'ont les gens de se divertir, d'être ensemble et de danser ». Totore rappelle également par ses propos que la danse ne peut s'apprendre avec des cassettes ${ }^{9}$, comme le font les jeunes aujourd'hui; elle ne peut se départir de son contexte de production et se comprend seulement à travers les conduites qu'elle suscite et qui lui donnent un sens. Ces danses, il a appris à les maîtriser presque toutes - plus d'une cinquantaine au total - en se montrant toujours très attentif à leurs diverses particularités ${ }^{10}$. En écoutant Totore, on sait toujours où l'on est : à Desulo (les basses sont plus liées et les formules nombreuses et brillantes), à Villanova Monteleone (où règne un étrange jeu rythmique exécuté au soufflet), à Mamoiada (le pasu torrau est censé venir de là et la version de Totore est si parfaite qu'aucun instrumentiste local n'oserait se mesurer à lui). Dans le ballu in piazza, cependant, il s'arroge volontiers le droit de mélanger les styles et de composer des suites de danses. Tout est "ballu sardu », mais, pour quelques experts, ces suites sont un passionnant voyage à travers les différents villages de Sardaigne. Totore, quant à lui, ne dit jamais, au cours de nos conversations privées notamment, qu'il emprunte à Mamoiada, à Oliena, à Desulo (trois villages importants du centre. Il cite Tore Pio, Francesco Maricosu, Bengasi, c'est-à-dire les musiciens de ces villages, avec qui il entretient des rapports en principe cordiaux mais où la jalousie ne fait jamais défaut. Et, en même temps que s'étend son savoir, se développe

Cahiers d'ethnomusicologie, 15 | 2002 
chez lui une véritable compétence d'analyste qui s'exerce sur ce qu'il voit (les microtraditions locales), autant que sur ce qu'il entend, joue et se plaît même, de temps en temps, à transcrire sur du papier à musique ${ }^{11}$.

La troisième période est celle de l'« artiste ": un mot nouveau que j'entendis la première fois dans sa bouche au début des années quatre-vingt-dix et dont l'emploi semble répondre à un nouvel esprit de liberté. A dire vrai, l'attention particulièrement aiguisée que Totore porte aux différentes musiques de Sardaigne l'a toujours tenu loin (jusqu'à ce jour) des musiques syncrétiques et «World». La musique sarde, dit-il, doit rester « intacte ». Pas question pour lui de participer aux messes fusionnelles qui, à l'initiative de jazzmen plus ou moins talentueux, se célèbrent désormais un peu partout en Sardaigne. Parfois pourtant on l'y convie. - «Sans doute, je ferai un jour un disque 'à moi' [i.e. de pure création], mais alors celui-ci n'aura rien à voir avec la Sardaigne "... «Chaque chose doit être à sa place »... « Ce que nous avons chez nous doit être gardé, il faudrait le mettre dans un coffre-fort et que personne ne s'amuse à le détruire, comme cela se fait déjà » [la liste nominative sur laquelle Totore et moi tombons d'accord ne mérite pas d'être mentionnée].

Cette même attention, il l'a longtemps manifestée à l'égard du costume. D'où qu'il provienne, celui-ci doit être uniforme, comme le reflet de la communauté villageoise qu'il représente et qui, théoriquement, doit être unique et unie. Or à Irgoli on n'en est plus là.

\section{...Et bientôt un quatrième}

Durant ces moments douloureux que traverse Irgoli, Totore, d'esprit moderne, travaille toujours sur la difficile nécessité de rendre conciliables des exigences contradictoires: expression traditionnelle versus "artistique", coutume et modernisme, mémoire et invention, etc. Mais, ce qu'il faut comprendre c'est que, pour ce qui est de la «sardité », il n'a de leçon à recevoir de personne. Il le dit, du reste: n'est-il pas, chaque semaine encore, sur les routes, dans toutes les localités de Sardaigne pour continuer de donner vie au ballu sardu ? Ne connaitt-il pas tous les villages un par un, leurs danses, leurs usages, la qualité de leur hospitalité respective ?...

C'est alors qu'il prend une initiative dans son village même, car toutes les initiatives de Totore ont Irgoli pour origine: celle de créer non pas une danse, mais l'expression chorégraphique d'une danse, en décidant de monter, au sein de son groupe, une sorte de petite compagnie de danseurs du village, composée d'hommes seulement, habillés non plus en costume traditionnel, mais à la façon des bergers des années cinquante: casquette, costume de velours, veste et gilet, guêtres en cuir lacées, grosses chaussures de campagne $^{12}$. Ce costume est fabriqué localement, par un excellent tailleur qui a également pour nom [To]tore, ce qui, désormais, ne devrait plus surprendre le lecteur.

Portée par une très grande énergie, cette nouvelle chorégraphie rencontre un vif succès (sauf auprès de certaines femmes qui trouvent anormal de s'en voir exclues). A l'évidence, elle rappelle avec efficacité qu'Irgoli est un pays de bergers - ou plutôt de pastori car le concept de pastore va bien au-delà du simple métier et renvoie à toute une culture encore très vivante et forte. Chacun sait là-bas que le cycle de violence et de vengeance enclenché depuis quelques années s'arrêtera bien un jour, mais surtout qu'il prend racine dans la cultura antica (antique) du pays : celle des pastori ${ }^{13}$. C'est eux que célèbrent Totore et sa nouvelle chorégraphie. En faisant endosser à «ses" danseurs, de façon 
spectaculaire, le vêtement des bergers (tel qu'il était porté il y a quelques décennies), Totore entend signifier par là qu'il convient d'assumer leur guerre fratricide, sans doute pour mieux exorciser le mal qui court dans tout le pays.

Pour ce qui est du costume et de sa fonction dans la représentation de la tradition, la position de Totore a donc changé. Ce costume - qui fait partie de l'identité même de tout gruppo folk - «il peut rester dans les armoires », dit-il aujourd'hui... « Il sera toujours le costume traditionnel et va bene!». Bref, s'il fait partie de la tradition, il n'est plus la tradition.

Cette nouvelle position m'intrigue, et je lui demande, en novembre 2001 :

- « Mais alors, Totore, c'est quoi, pour toi, la tradition ? » [question posée chez lui, alors qu'il est en train de rafistoler sa nouvelle et imposante maison. Pour une fois, je pense à enregistrer la réponse].

- « Pour moi, cela ne consiste pas à laisser intacte une chose sous la forme qui a toujours été la sienne ou que nous avons toujours connue. C'est d'abord une espèce de magie ( magia) qui permet aux gens de pouvoir passer du temps ensemble à s'amuser (divertirsi). Ce n'est pas le costume, ni la danse elle-même (ou la danse toute seule) qui en est la base. C'est d'abord et surtout une façon d'être ensemble ».

- «Indépendamment de tout contenu ? Selon toi, être ensemble, comme tu le dis, devant la télévision pour regarder un match de football relève de la tradition? »

-Oui, oui...

40 Au moment de transcrire ce passage, je mesure la pauvreté de l'écrit par rapport à l'oral. Le « oui » de Totore ne renvoie pas à ma question mais au fait qu'il a compris l'objection. Visiblement, cette objection, il se l'était déjà faite. C'est un "oui » qu'il s'adresse à luimême. Je m'arrête en revanche sur un autre mot nouveau de son vocabulaire: celui de magia. Ce mot, je l'avais entendu déjà dans la bouche du Tenore de Bitti - le chœur de loin le plus connu de toute la Sardaigne et qui tourne sans cesse dans toutes les capitales d'Europe, d'Amérique et d'Asie. Mais c'est une expression qui court aussi beaucoup dans les médias italiens (un mot d'une grande vulgarité, donc), où l'on parle de la « magie d'un spectacle » lorsqu'on ne sait pas trop quoi en dire.

41 La magie dont parle Totore est d'une nature sensiblement différente et, sans doute, plus fidèle à l'étymologie du mot. Il sait que l'on peut créer des relations communautaires quasi « magiques » à partir de choses très simples, en les faisant sortir comme le lapin du chapeau d'un prestidigitateur, à partir de rien ou presque: de quelques formules d'accordéon, bien sûr, mais tout autant "d'un bout de pane e formaggio », comme il le dit lui-même un peu plus tard dans l'enregistrement. Lui et sa musique ne sont que l'opérateur de cette magie. Mais, toute magie a sa technique et, contrairement à ce que l'on pourrait croire, la sienne n'est pas seulement musicale. Elle repose sur une attitude et un comportement, et notamment une façon d'être avec les autres. En fait, c'est l'homme autant que le musicien qu'on invite sur toutes les places de Sardaigne. Car la Sardaigne ne manque pas d'accordéonistes - et certains jouent à peu près aussi bien que Totore. Beaucoup, en tout cas, l'imitent ou cherchent à l'imiter - et bien sûr visent à le surpasser. Reconnaissons que, de nos jours, il n'est pas toujours facile de savoir, à l'écoute d'un enregistrement, si c'est Totore qui joue ou un jeune confrère. Mais, in vivo, il n'y a pas à se tromper, car Totore ne se rend pas à une fête seulement pour jouer, mais pour être luimême, apporter de nouvelles barzellette (pas toujours drôles du reste, mais qui servent surtout à créer un terrain d'intercompréhension entre lui et ceux à qui il les adresse). Il 
sait surtout se montrer attentif aux autres, c'est-à-dire aux affaires du pays et à ce qui se passe « derrière la scène ». En Sardaigne, la tradition passe encore par là, plus que par le disque et le spectacle.

42 En résumé, la carrière de Totore se laisse appréhender à partir de quatre mots-clés, chacun d'entre eux représentant une étape :

- Village (paese)

- Sardegna (Sardegna)

- Artiste (artista)

- Magie (magia).

Successivement, c'est d'une petite unité territoriale qu'il est question, puis d'une entité régionale. Vient ensuite l'affirmation de la personnalité du musicien assumant pleinement la charge de reproduire, d'entretenir, voire de recréer sa propre culture au contact étroit de ceux qui la partagent. Une culture que la société moderne fragilise mais qu'à ce jour, elle n'a pas réussi à détruire complètement.

\section{Totore et la Sardaigne : un cas de « parallelismo convergente »}

Mais la carrière de Totore ne tient pas seulement à sa personnalité. Elle doit beaucoup à l'histoire récente de la Sardaigne. Il le reconnaît lui-même : l'âge de sa maturité coincide avec l'éclosion des gruppi folk, qui ne toucha pas seulement Irgoli, mais toute l'île. Cette éclosion relève elle-même d'un revivalisme qui affecta toute l'Europe occidentale, mais qui prit une forme assez originale en Sardaigne. Toujours est-il qu'en quelques années, chaque village sarde ou presque se trouva pourvu d'un gruppo - et bien souvent de deux, pour la raison mentionnée à la note 7. C'est par l'intermédiaire de ces gruppi, émanation directe d'un savoir-faire strictement villageois et assumant un rôle de représentation ${ }^{14}$, que la musique locale et populaire connut un très fort développement. Sur place, toutes les fêtes s'ouvrent à leur présence ; partout on les invite pour qu'ils se produisent... et la piazza devient spectacle. Aujourd'hui plus que jamais, il est inconcevable de se passer d'eux. En d'autres termes, les fêtes devinrent progressivement des lieux d'exhibition et de technicité choréografico-musicale. Comme il fallait faire danser tous ces gens-là, Totore ne manqua pas de travail. Et tandis que les villages se parent de leurs plus beaux costumes et apprennent à se connaître par l'intermédiaire de leurs gruppi, l'accordéon prend une importance et une autonomie qu'il n'avait pas eue auparavant.

Cette vertigineuse montée en puissance des gruppi fut une véritable révolution culturelle en Sardaigne. Certes, auparavant, des contacts existaient entre les villages, mais ils étaient sporadiques et surtout n'avaient aucun caractère institutionnel ${ }^{15}$. Jusqu'aux années soixante, un musicien avait normalement pour rayon d'action quelques localités voisines de chez lui, situées à peu de distance les unes des autres. De sorte que si Totore était né quelque cinquante ans plus tôt, il est probable qu'il serait resté suonatore de son pays et que personne - pas même sa mère - n'aurait éprouvé le besoin de lui enlever le diminutif que, durant sa prime jeunesse, on accola à son nom : il l'aurait gardé, comme l'avait fait, jusqu'à la fin de sa vie, son modeste et excellent prédécesseur «ziu Tonareddu $»$. 


\section{NOTES}

1. Cette année, ce chiffre est passé à six mille, à la suite de la dramatique «maladie de la langue bleue ", dont on a peu parlé en Europe du Nord, mais qui a littéralement décimé les troupeaux ovins de Sardaigne, et qui toucha également la Corse.

2. Parfois, profitant du jeu de mot que permet l'italien, on lui donne aussi - et comme par luxe - le surnom de suonato-Tore (dérivé du mot "suonatore» qui signifie "musicieninstrumentiste ». La précision ethnograpique m'oblige à ajouter que le diminutif Totore concerne plutôt la partie orientale du centre de la Sardaigne. Au centre-centre de l'île, en Barbagia, Salvatore donne lieu volontiers à d'autres dérivations (Barore, Bore, etc.).

3. En fait, l'affaire est peu plus compliquée que ce que j'en dis car, dans un village voisin, Totore a un cousin, accordéoniste lui aussi - un parfait homonyme puisqu'il s'appelle également Totore Chessa. Celui-là, on l'appelle parfois «l'autre Totore». Mais, en dépit de cette ressemblance formelle, il n'y a guère de confusion possible - cet « autre » est un pâle reflet de celui qui nous concerne et l'adjectif indéfini, lorsqu'il est utilisé, a toujours une connotation négative.

4. A dire vrai, ce costume n'est pas aussi «old fashioned» et artificiel qu'on pourrait croire puisqu'il était régulièrement porté jusqu'au début de ce siècle et parfois même plus tard. Parfois, il poursuit sa vie sous sa forme originelle au fond d'une armoire. Mais le plus souvent, il est refait à partir de ce modèle par les femmes de la famille et pour un usage strictement familial.

5. «Pays » est ici la traduction de « paese » qui renvoie, comme autrefois en provençal, au village et non à une aire géographique.

6. Je tiens cette métaphore, ainsi que quelques autres, d'une interview de Totore que Fabrizio Giuffrida a réalisé pour la revue italienne Cous cous, janv.-févr. 2001 : 16-19.

7. Un mécanisme duel, en ce sens qu'il implique toujours deux parties. Au titre de ce mécanisme, citons la vendetta contractuelle (entre deux familles), la compétition à deux, très développée sous toutes ses formes et qui touche aussi bien la poésie que la pratique du cheval, de la danse, du chant à guitare et du jeu de mourre (en sarde : sa murra). Il s'agit d'un jeu de hasard fondé sur un calcul de probabilités dans lequel deux hommes se montrent rapidement et simultanément un certain nombre de doigts dressés en criant un chiffre supposé représenter la somme des doigts déployés. Il s'agit en fait d'un jeu d'habileté car, pour l'emporter en donnant le chiffre juste, il convient de deviner très rapidement les stratégies de l'adversaire tout en prenant soin de dissimuler les siennes. Plus largement, la société sarde se mesure toujours à travers un jeu de compétition de nature aussi bien rituelle que profane. Et il est rare de trouver une initiative locale, quelle qu'elle soit, qui ne donne lieu à une contre-initiative directement concurrente. A Irgoli, par exemple, s'est développée très récemment une nouvelle passion - le pays est attachant en cela car il ne se lasse jamais de se mobiliser sur des choses nouvelles: le chant religieux. L'année de cette découverte - ou plutôt de cette re-découverte, il y a cinq ans - a été aussi l'année de la création non pas d'un groupe de chanteurs, mais de deux, concurrents. Depuis deux ans... c'est le base ball féminin (dit, paraît-il, « soft ball »). Les jeunes femmes d'Irgoli sont allées jusqu'en finale, où elles ont perdu contre l'équipe de Rome!

8. En automne 2001, je reviens avec Totore sur cette initiative et sur cette usurpation. Il la regrette me dit-il. "Toi et Francesco, vous aviez raison!", reconnaît-il. A leur façon, nos compétitions oratoires et conceptuelles sont bien sardes. Cette fois, c'est moi qui marque un point. Je le lui dis, d'ailleurs.

9. Ces cassettes, d'ailleurs peu onéreuses, se vendent depuis longtemps sur les marchés, où elles sont présentées dans des bacs, mélangées avec d'autres musiques légères italiennes. 
10. Par ailleurs, par souci patrimonial, il se constitue une belle collection d'accordéons anciens, collectés en Sardaigne même, mais aussi en Italie continentale et à l'étranger durant ses voyages. 11. Bien qu'assez grossières, ces transcriptions sont correctes. Totore, il y a deux ans, m'en offrit une, significativement présentée dans un cadre, comme s'il s'agissait d'un tableau de maître.

12. De ce costume, restent en usage aujourd'hui, outre les grosses chaussures, le pantalon (porté très serré), parfois la veste et la petite casquette.

13. Il y a à Irgoli plus de cent cinquante bergers, le plus souvent regroupés en coopérative. Ils sont très visibles et volontiers bruyants à l'intérieur d'un village où, à l'évidence, ils occupent une place sociale et économique centrale

14. Dans les deux sens du terme : représentation spectaculaire en direction des autres, en même temps que représentation de soi $-c f$. le rôle essentiel du costume à fonction fortement identitaire.

15. Si ce n'est très localement. Ces contacts avaient lieu soit par l'intermédiaire de sanctuaires à vocation micro-régionale, communs à plusieurs villages et fréquentés plusieurs jours de l'année durant les feste lunghe [fêtes longues et/ou neuvaines]; soit, à une autre échelle et pour un autre contenu, à l'occasion de la Festa del Redentore de Nuoro qui, depuis très longtemps, est un lieu de forte agrégation villageois, donnant lieu à des manifestations importantes: initialement des processions qui se sont transformées en « sfilate» - en défilés folkloriques.

\section{RÉSUMÉS}

L'article se propose de suivre le parcours idéologique et musical de Totore Chessa, un accordéoniste sarde encore jeune et désormais célèbre dans toute l'île. Sa forte personnalité est prise comme fil conducteur pour comprendre la dynamique culturelle des villages de Sardaigne centrale et l'évolution de la musique durant ces trente dernières années : - 70: explosion des gruppi Folk, mouvement revivaliste, n'impliquant pas une rupture nette par rapport aux traditions des décennies précédentes, mais ouvrant les villages à une intercommunication musicale ; -80 : scission du gruppo Folk d'Irgoli (village de Totore) et autonomisation de Totore qui devient non plus le musicien de village qu'il était, mais l'accordéoniste de (presque) tous les villages de Sardaigne:- 90: professionnalisation complète de Totore et introduction de chorégraphies «stylisées». Chacune de ces étapes s'accompagne chez notre ami-musicien de l'élaboration dun nouveau discours et d'un glissement de vocabulaire dont les maîtres-mots sont successivement «Paese [Village]», "Artista» et "Magia»- une «magie» qui renvoie paradoxalement à une conception moderne de la tradition.

\section{AUTEUR}

\section{BERNARD LORTAT-JACOB}

Bernard LORTAT-JACOB est Directeur de recherche au CNRS, responsable du Laboratoire d'ethnomusicologie du Musée de l'Homme. Il travaille depuis maintenant plus de trente ans sur les traditions musicales européennes et méditerranéennes (Maroc, Sardaigne, Roumanie, Albanie) et a publié de nombreux disques et articles, ainsi qu'une dizaine de livres dépassant souvent le cadre de la stricte monographie. 